\title{
Implementing a modified early warning system for critically ill patients in an acute hospital
}

\author{
Michael Page ${ }^{1}$ RN; MRes, DipHE, Clinical Nurse Specialist, \\ Ian Blaber ${ }^{1}$ RN; DipHE, GradDip (Coronary Care Nursing), Clinical Nurse Specialist, \\ Patricia Snowden ${ }^{1}$ RN, Associate Professor, Director of Nursing. \\ ${ }^{1}$ Mater Private Hospital, Brisbane, Australia.
}

E-mail: michael.page@mater.org.au

Key Words: critical illness * modified early warning system * response algorithm

\section{SUMMARY}

- This paper describes a project to implement an early warning scoring system within an Australian private hospital.

- Sub-optimal care of critically ill patients on general wards has been described in the literature. Early warning systems have proved successful in supporting ward nurses to respond to deteriorating patients. They have been used mainly in the UK and in the public health system.

- We believed that a modified early warning system could be adapted to the specific needs of an acute private hospital and would also have a positive effect on the outcomes of critically ill ward patients, and on the confidence of ward nurses caring for them.

- The aim of the project was to pilot a nursing tool, comprising a colour-coded observation chart and response algorithm, to support ward nurses in the early identification of, and rapid response to deteriorating patients on two general wards.

- The setting for the study was a tertiary, acute private hospital in Brisbane, with 323 beds. The pilot study was carried out on a 30-bed neurovascular ward and 41-bed orthopaedic ward from October to December 2007.

- Implementation of the new system improved clinical outcomes for patients on the pilot wards. Compliance with documentation was very high and nurses' satisfaction with all aspects of the new system was extremely high. The modified early warning system was considered a valid tool, valuable in supporting ward nurses in the care of critically ill patients and the system was extended to all hospital wards after the pilot.

\section{INTRODUCTION}

Sub-optimal treatment is common in the period before an intensive care unit (ICU) admission from general wards, and is associated with increased ICU and hospital mortality (Goldhill, 2001; MacQuillan et al., 1998; McGloin et al., 1999). Patients admitted from general wards to ICU have higher mortality rates than those admitted from emergency or operating departments, and around $30 \%$ of patients admitted to ICU from a ward have suffered cardio-respiratory arrest (Goldhill 2001; Sprung et al., 1999; Metcalfe et al., 1997). Patients discharged from ICU to general wards often experience sub-optimal care in areas where nurses are too busy or lack the critical care skills to identify and respond appropriately to a deteriorating patient (Wallis et al., 1997). Half of post-operative deaths occur on wards, and the majority after more than five days post-surgery (Campling et al., 1995; Lunn et al., 1996).

Many patients who deteriorate on general wards do so over a protracted period; they do not just suddenly arrest. In many cases, retrospective case-note analysis shows abnormal physiological measurements leading up to the arrest team being called, without an adequate response to the patient's changing condition (Wood \& Smith, 1999). This may potentially be a consequence of increasingly acute patients being admitted to general wards, with constraints on staffing, and potentially inadequate skill-mix to care for patients with critical care needs.

Goldhill (2001) points out that while all acute hospitals have cardiac arrest teams they are relatively ineffective and expensive, and avoid the real problem. This leads to an $80 \%$ mortality rate for the relatively small number of patients who arrest on the wards and survive long enough to be admitted to ICU, compared to $44 \%$ for all other admissions from wards (Goldhill, 2001; Goldhill \& Sumner, 1998).

In response to this sub-optimal management of sick patients, medical emergency teams (METs) have been established to respond to patients with certain abnormal physiological measurements. While METs were pioneered in Australia (Lee et al., 1995) it was in the United Kingdom (UK) that this service was further developed into patient at risk teams (PARTs), which reduced the number of arrests on wards and led to earlier ICU admissions for some patients (Hadfield et al., 2000).

The introduction of such teams reduced the number of unexpected cardiac arrests by $50 \%$ (Buist et al., 2002), yet in most cases, they provide a response to emergencies only. In the UK, after the publication of Comprehensive Critical Care (Department of Health, 2000), intensive care outreach services were established in most acute UK hospitals to delay or prevent the need for emergency response to a deteriorating ward patient (Goldhill 2001).

The UK Department of Health (2000) recommended that critical 
care services should be provided, not only for those patients who are critically ill, but also for patients at risk of critical illness, whether in ICU or the general wards. This was conceptualised as 'critical care without walls'.

Sick patients on the ward need early identification and prompt and appropriate treatment. A number of factors have been identified which often leads to a series of events where deteriorating patients are treated sub-optimally. The result is that nurses often fail to call for medical assistance as patients deteriorate (Schein et al., 1990; Franklin \& Matthew, 1994). The main causes are claimed by McQuillan et al. (1998) to be, "A failure of organisation, lack of knowledge, lack of supervision, and failure to seek advice."

Early warning systems (EWS) addressed such criticisms and are based upon the allocation of a points score to physiological observations, the calculation of a total score, and designation of an agreed calling 'trigger.' According to Parissopoulos and Kotzabassaki (2005), the use of physiological 'track and trigger' tools provide "Timely recognition of all patients with potential or established critical illness regardless of their location, and timely attendance to all such patients, once identified, by those possessing appropriate skills, knowledge and experience."

The original EWS (Morgan et al., 1997) was based on five physiological parameters: systolic blood pressure, pulse rate, respiratory rate, temperature and AVPU (conscious level) score (Subbe et al., 2001). The EWS has been modified to incorporate different physiological parameters in different settings, and has been validated for use in both surgical wards (Odell et al., 2002, Stenhouse et al., 2000) and medical wards (Subbe et al., 2001). Its purpose is to provide an aggregate score generated from baseline recordings of vital signs (the greater the deviation from normal parameters, the higher the point scores). Clinical deterioration is detected and medical intervention can be implemented at an early stage in the patient's illness.

However, any type of physiological tracking and triggering tool should be accompanied by an algorithm, which is the key element in ensuring an early response from medical staff or the PART (Parissopoulos \& Kotzabassaki, 2005). A trigger score means that the patient needs to be reviewed by the appropriate staff urgently (Featherstone et al., 2002).

\section{DEVELOPING THE MATER PRIVATE MODIFIED EARLY WARNING SYSTEM}

The post of Clinical Nurse Specialist (CNS) was introduced at the Mater Private Hospital as a supportive move for wards after hours, that is, the period outside the normal 0800-1700 day, in what has been recognised as an increasingly technical post-operative environment. The CNS role supports ward nurses in early detection and enables prompt intervention to avert deterioration of patients.

Following case note review by the CNS of a number of MET calls, it was discovered that often patients' physiological observations demonstrated gradual deviation from normal physiological observations; as has been noted in the literature. Physiological parameters, in some cases, had been outside normal limits for as much as 48 hours preceding the MET call, but no single reason for missing this gradual deterioration could be identified. Therefore, we decided to carry out a literature review and to identify the best elements of existing MEWS for use in the specific environment of an acute private hospital.

Between April and September 2007 the two CNSs, with support from the Director of Nursing, developed a MEWS, with the following aims:

- to empower ward nurses to make optimal decisions about at risk patients;
- to improve multi-disciplinary communication, particularly afterhours; and

- to reduce inappropriate MET calls and delay deterioration that precipitates a MET.

The primary endpoint was to reduce medical emergencies by empowering the nursing staff to recognise all patients with potential or established critical illness, and respond by promptly initiating appropriate and optimal clinical intervention.

We began by searching the evidence, using database searches of the Cochrane and Joanna Briggs Institute databases of systematic reviews; Medline (1966-2007); CINAHL; Pubmed; and Google Scholar. Search terms included: early warning system*, early warning scor ${ }^{*}$, track and trigger, critical care outreach; intensive care outreach. This generated a body of literature outlining the development of METs and MEWS, initially in Australia, but with gathering momentum in the UK and USA

It was considered vital not to 'reinvent the wheel' but the vast majority of papers on MEWS were published in public hospitals (most notably in the UK National Health Service) and were not necessarily directly transferable to an Australian acute private hospital. Our aim was to take the best of what was currently in use and modify it to our specific needs.

Our existing observation charts had not changed much over many years, and did not appear to be evidence-based. They were used to record only four-hourly physiological measurements, which may be adequate for many ward patients but are completely inappropriate for others, and visually could not detect physiological trends.

We wanted to design an observation chart that was easy to understand and complete, which did not produce extra work for the ward nurse. Choice of layout was loosely based on one in use at Ysbyty Glan Clywyd Hospital, Wales, UK (Institute for Healthcare Improvement, 2007), which we agreed had the most visual impact. Its colour-coded warning areas made it impossible to ignore a patient's deterioration, countering issues of untrained nurses failing to report scores, which trigger a response to the senior nurse (Palmer, 2004). Action required is, with education, indisputable.

The first draft Mater Private Modified Early Warning system and algorithm (see figures 1 and 2) explain clearly what to do should the patient's physiological measurements indicate deterioration. If they move into the yellow area, a MEWS score must be recorded, and the corresponding action taken, using the accompanying algorithm (figure 2).

\section{Scoring}

A MEWS score of 1 would suggest the patient is showing signs that cannot be ignored, but which at this stage do not necessarily merit a MET call or Visiting Medical Officer (VMO) call. However, if these clinical indicators are missed or ignored the patient could continue on a downward trajectory and eventually require emergency assistance later. Therefore, a MEWS score of 1 prompts a clear and concise response. In most cases this simple action will lead to the patient being stabilised or returning to within normal parameters. However, should the patient deteriorate, their observations are recorded more frequently and there will be less chance that a change in status will be missed or fail to create a response.

If the patient's MEWS score is 2 or 3 the frequency of observations is increased further. The CNS must be called, and should attend promptly. After CNS review of the patient the VMO must be informed. At this stage, it is possible that optimising management of the patient (for example, positioning, pain management, electrocardiogram recording and/or telemetry, fluid delivery) will prevent deterioration. In addition, the CNS will be able to order blood tests and other investigations (if necessary) before calling the $\mathrm{VMO}$, so the full picture 


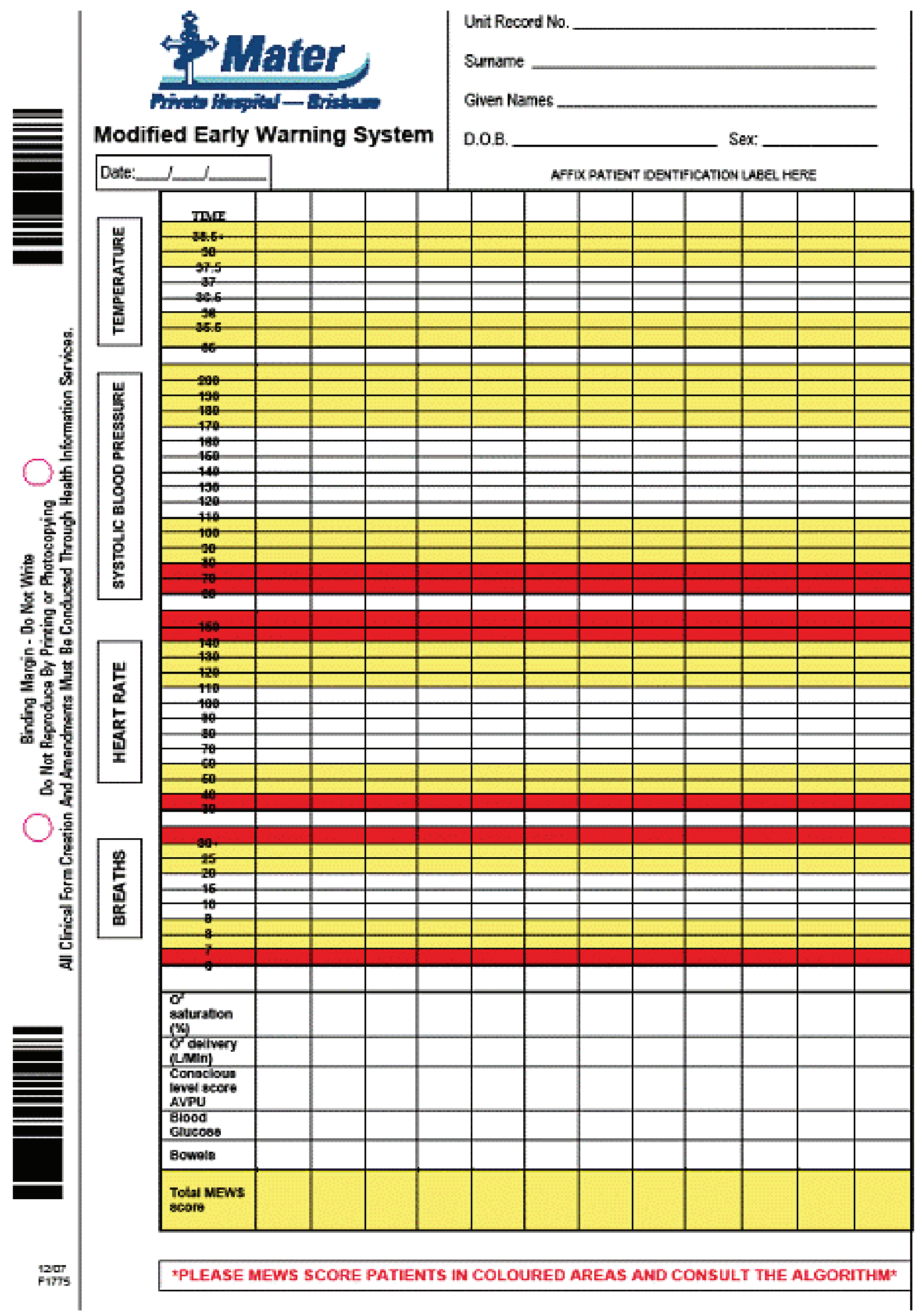




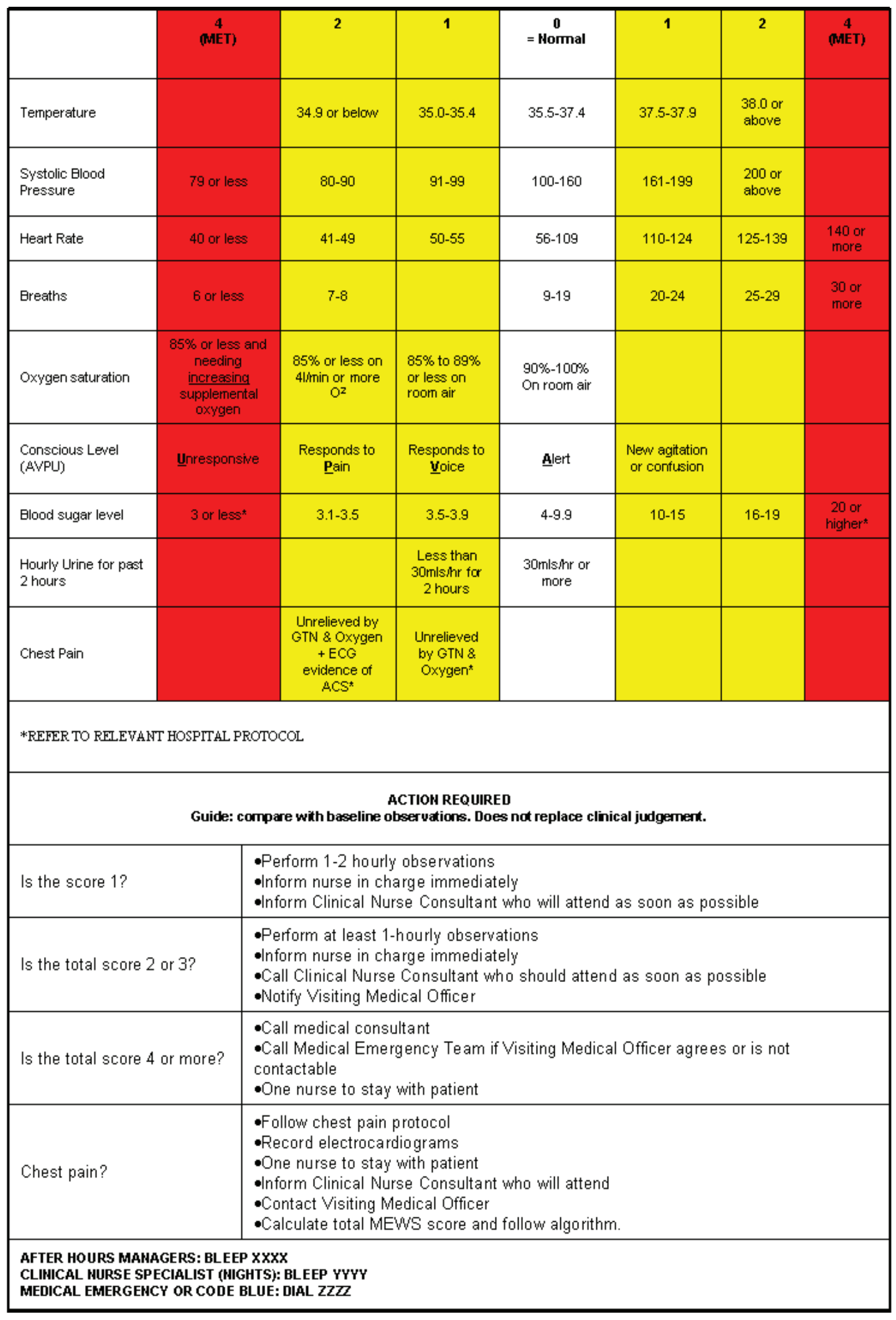


of the patient's condition is available when the VMO is advised. If the patient's cumulative MEWS score is 4 or more, or the red area is entered, urgent action is needed. The parameters follow existing MET criteria and require immediate notification to the VMO and then a MET call (if not contraindicated by the VMO). The CNS will arrive as part of the MET but in most situations will already be aware of the patient's history as in many cases patients will trigger a response in the yellow area before entering the red. Only occasionally will a patient unexpectedly deteriorate rapidly from normal parameters to the red area, requiring emergency treatment. This has been noted in the literature; catastrophic deterioration is frequently preceded by documented deterioration of physiological parameters (Sax \& Charlson, 1987; Smith \& Wood, 1998, Cited in Subbe et al., 2001).

\section{The algorithm}

The algorithm does not replace clinical judgement but does make procrastination difficult. It relies on accurate measurement of physiological measurements and correct documentation, both of which are education and training issues. Its effect is to empower nurses to make evidence-based decisions and to seek appropriate support. That support is intended to be a resource and presents a further opportunity for coaching the ward nurse rather than taking over the management of the patient or deskilling the nurse. The CNS role is one of support, advice and coaching.

\section{Working with the MET}

At Mater Private, the MET team is staffed by the critical care medical officer (CCMO) on duty in ICU. This means that for every MET call the $\mathrm{CCMO}$ is taken away from patient care in ICU. This represents a huge time burden for CCMOs, especially as they are often unfamiliar with the history of the patients they are urgently asked to review. In many cases, the calls were made by junior nurses unaware of the clinical picture, and CCMOs felt such calls were an onerous workload. The MEWS further modified the MET provision while protecting existing criteria, which were valid and workable. It added another safety layer of assessment, which would reduce inappropriate calls and improve the clinical outcome of patients, negating the circumstances leading to a MET trigger.

\section{Clinical judgement}

The CNS is able to make clinical judgements about whether to call CCMOs in ICU for advice, to initiate nursing interventions to stabilise the patient, or call a VMO for further advice. The MEWS assists this by prompting ward nurses to refer patients of concern to the CNS after hours, that is, any patient with a MEWS score of 1 or greater, and any patient without a MEWS trigger who the nurse has concerns about.

The CNS will become aware of all patients who have moved into the yellow area of the MEWS chart and be able to advise ward nurses of the optimal response. This may include actions such as increasing frequency of observations, repositioning patient, administering effective pain relief, reassuring the patient, or ensuring adequate hydration. However, if a patient's MEWS score increases it will allow further timely intervention. If the patient's MEWS increases to the point where a VMO is called, the ward nurse or CNS will already have important clinical and biochemical data at hand to inform the VMO.

\section{THE MEWS PILOT STUDY}

\section{Education issues}

While the new chart is not an enormous change from that used previously, it required advanced training and discussion with the nurses who would use it, so that it would be used accurately and completely, and the reasons for its use were established.

Initial reaction to the MEWS was positive, as it was clear and easy to use, yet provided valuable information and guidance for clinical decision-making. Nurses voiced that it would not create more work for them, but should ease their workload as students and less qualified/experienced nurses could be left to record the observations with clear guidance on when to report abnormalities to the senior nurse.

The MEWS provided an opportunity for nurse educators to implement a consistent programme of basic assessment skills in areas such as breathing, airway maintenance, haemodynamic status, renal status, administration of oxygen, and response to chest pain. Change then presented both a threat and an opportunity to the ward nurse, but from a quality viewpoint it allowed us to standardise our knowledge base at a higher level and change misconceptions and ritualistic nursing practices that are known to exist in the profession (Walsh \& Ford, 1989).

Education took the form of a cascade, starting with the CNS to the after hours managers, down to the nurse educators for each ward, who then worked with the nurse unit managers and Level 2 nurses, and finally to the Level 1 nurses and then the nursing students and non-qualified nurses.

\section{Consultation with stakeholders}

We arranged meetings with the nurse unit managers of the wards we believed would be ideal to pilot the project, and the nurse educators responsible for those wards, to discuss the project and to elicit any fears or concerns they might have had. At the same time an ICU CCMO was able to provide audit data on the quantity and triggers of MET calls over the past three years, and to feedback on the validity of the parameters we set, and the consistency with existing hospital protocols.

The nurse educators arranged formal and informal education events on the use of the MEWS, and a visual presentation was loaded onto the computer in the wards piloting the MEWS. Initial, as well as ongoing, education was seen as a key to the ultimate success of the pilot.

It was considered essential to implement the new system in a number of steps, and to evaluate its use at each stage. Previous authors have noted scepticism and resistance to change (Trevett, 2007; Institute for Healthcare Improvement, 2007) and while we expected to reduce the workload and stress of ward nurses, some difficulties in implementation were inevitable.

\section{The pilot wards}

We carried out a pilot of the MEWS on a 30-bed neurovascular ward (9 East) and a 41-bed orthopaedic ward (8 South) between October and December 2007 (8 weeks). A programme of education and awareness of the MEWS meant that the management and nurses on the ward were eager to be the pioneers of the system in the hospital. Weekly spot audits demonstrated an extremely high compliance rate of greater than $80 \%$ (range $82.5-97.5 \%$ ) in all audited categories, and an averaged overall compliance of $89.8 \%$ (9 East) and $92.3 \%$ (8 South) over the pilot period (see figure 3 ).

\section{DISCUSSION}

Many UK hospitals report evidence of the benefit of track and trigger warning systems in improving care of the critically ill patient. A key question for this pilot study was whether similar benefits could be demonstrated in an Australian private hospital. Following the pilot, we audited the effectiveness of the MEWS in order to modify aspects to improve its ease of use and value for clinical decision-making. 


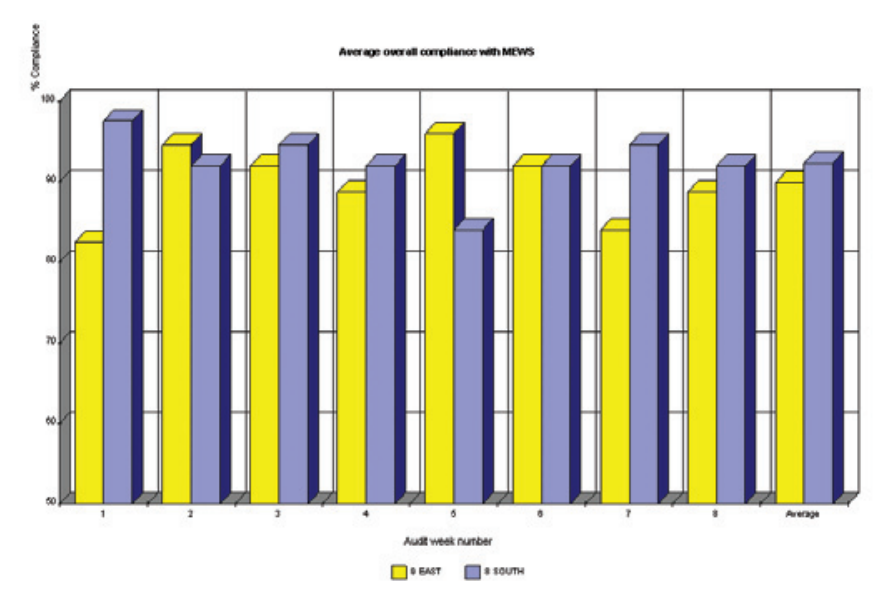

Figure 3: Compliance with MEWS documentation

Audit indicators based on benefits of MEWS reported in the literature were:

- Did the MEWS increase satisfaction/confidence of nurses in caring for critically ill patients?

- Was there increased satisfaction amongst VMOs/ICU doctors?

- Was there a reduction in number of medical emergencies on participating wards?

Nurses' attitudes

The new system was immediately popular amongst nurses on the pilot wards, as reflected in the exceptional documentation compliance. We confirmed the popularity and ease of use of the MEWS in a subsequent satisfaction survey of all regular nurses on the pilot wards (see figures 4 and 5). The survey also allowed nurses who would be using the chart and algorithm to make suggestions for changing it. These suggestions were incorporated into a revised version, but were minor additions (mainly adding elements that were removed from the old observation charts), such as the recording of bowel movements and body weight, as opposed to changes to the system itself.

Sixteen questions on all aspects of the MEWS were asked in a questionnaire sent to all regular nurses on the pilot wards. We achieved a satisfactory response rate of $66.6 \%(n=30)$. Most questions utilised a five-point Likert scale to assess nurse satisfaction with the use of the MEWS and its effect on patient care, safety, identification of, and response to, a deteriorating patient. Three open-ended questions allowed more qualitative feedback on the new system, and on the supporting CNS role.

Figure 4 shows responses that illustrate the degree to which nurses felt the new observation chart was better and figure 5 indicates how they rated it to detect patient deterioration. These results demonstrate that nurses found the new chart better, and believed that it had led to improved clinical outcomes for patients on the pilot wards.

The new system proved popular. The majority of respondents $(76.6 \%)$ stated that the MEWS was either better $(53.3 \%)$ or far better $(23.3 \%)$ than the existing observation charts. Ten percent believed it was about the same as existing documentation, $6.6 \%$ thought it was worse and $3.3 \%$ far worse. $3.3 \%$ gave no answer (figure 4 ). Respondents were similarly emphatic regarding the superiority of the MEWS over the previous charts in terms of safety and clinical outcomes for patients: $23.3 \% \%$ stated it improved care a great deal, $56.7 \%$ that it improved care, and the rest that it was about the same $(20 \%)$ as the old system. None thought it worse in this respect.

Figure 5 shows that the overwhelming majority of nurses $(87 \%)$ considered the MEWS either improved care a great deal $(26 \%)$, or



Figure 4. The MEWS compared with existing observation charts

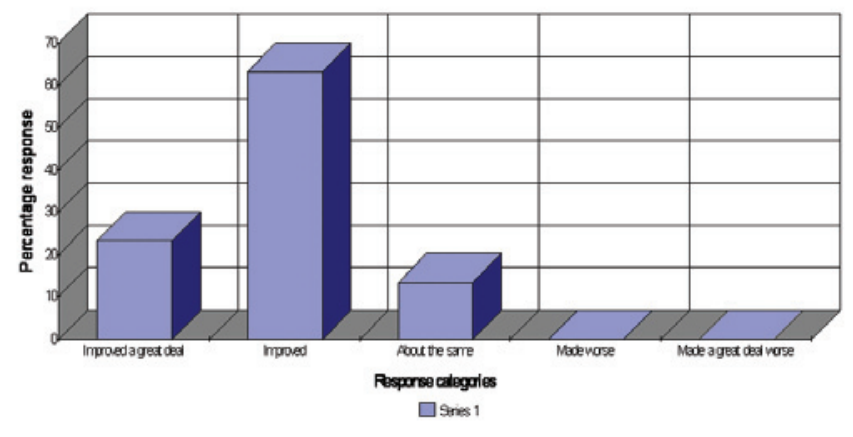

Figure 5. Early identification of a deteriorating patient

improved care $(61 \%)$, compared with the previous system, in terms of identification of a deteriorating patient. In terms of response to a deteriorating patient, a similarly high percentage claimed it improved care a great deal $(23.3 \%)$ or improved care $(63.3 \%)$, while the remainder believed it was about the same $(13 \%)$. These findings indicate the level of success of the pilot.

Further demonstration of the popularity of the MEWS on the pilot wards was evidenced in the responses to the open-ended questions: What do you like/dislike about the MEWS? Some typical responses were:

- "Have definitely noted this system to improve patient outcomes and quality of care."

- "It is visually easier to see the patients observations and any changes throughout the day."

- "Easy to notice when a patient's condition is deteriorating and what action to take."

- "Great! Helps nurses to prevent or reduce MET [calls] by initiating appropriate clinical intervention."

Negative comments were rare. However, one untypical reply, which summarised a nurse's dislike at having to calculate a MEWS score in order to trigger a response, is given below (although, even this comment was ultimately restrained):

"[lt is] time consuming adding scores and explaining to agency/casual staff how to use it (although [it is] self explanatory if [I] take the time to read [the] guide chart)."

A key theme from the qualitative comments was that nurses valued both the MEWS and the related support that was given from the CNS, especially after hours. Nurses on the pilot wards saw the MEWS and CNS role as inextricably linked. The following comment was typical:

- $\quad$ "MEWS picks up on initial worried phase regarding a patient, which may not necessitate calling a VMO, however we can still notify a senior member of staff."

While the MEWS seemed popular and valid, further work would be 
required to ascertain whether a MEWS alone is valuable or whether support in the form of a nurse-led service or medical officer is what is especially valued by the nurses.

\section{Doctors' attitudes}

While the MEWS represented a change to nursing rather than medical documentation, its effects would have consequences for response to patients and in particular, at what stage a medical emergency is called. Support for the pilot was evident from the ICU doctors who felt improvements to the existing system were possible. They welcomed opportunities to reduce their ward-based workload while retaining the safety valve of the existing MET provision. During the MEWS pilot we had no negative feedback from doctors and, similarly to the nurses, they saw the MEWS and CNS role as combined, and generally welcomed it.

A satisfaction survey was sent to a purposive sample of ten doctors (1 ICU intensivist, 3 CCMOs, and 6 VMOs). The VMO sample comprised two physicians, two orthopaedic surgeons and two neurovascular surgeons who regularly visited one or both the pilot wards. Their responses were sufficient to gauge doctors' attitudes without claiming representativeness. It was clear that the MEWS seemed to enhance consultation with VMOs, as they were less likely to be called spuriously (most noted a reduction the calls they received from the pilot wards), and when they were called the nurse's decision was underpinned by sound evidence that there was a potential problem for the patient. Doctors generally welcomed the track and trigger elements of the chart and the likelihood that changes to patient status would be notified to them at a stage when they could do something to prevent deterioration.

\section{Medical emergencies}

The number of medical emergencies recorded over a period of time is dynamic due to the multi-factorial nature of such decisions to call and seasonal trends such as skill mix, bed occupancy, number and complexity of operations, and availability of higher-level beds. However, after the introduction of a new hospital protocol for calling a MET in September 2006, calls from the wards had been reasonably stable at 2.9 per ward per month in the year leading up to the MEWS pilot $(n=246)$. Our pilot wards sat in the middle of the 'MET table' with 9 East calling 2.75 and 8 South 2.08 MET calls per month, on average. 9 East therefore contributed an average of $13.4 \%$ of hospital MET calls and 8 South $10.1 \%$ in the twelve months prior to the MEWS pilot.

During the MEWS pilot, average MET calls called per month on the pilot wards fell significantly on 9 East from 2.75 to 1.5 per month (and from $13.4 \%$ of total hospital MET calls to $8.3 \%$ ) and on 8 South from 2.08 to 1.5 per month (and from $10.1 \%$ of total hospital MET calls to $8.3 \%$ ). MET calls on the five wards not using the MEWS remained static during the same period (up from 2.9 to 3.00 per month) (see figure 6).

While we tentatively report a reduction in MET calls because of the pilot, further analysis of the effect over an extended time period needs to be carried out to be able to make statistically significant claims. Anecdotal evidence from the pilot wards supports the claim that MET calls have been averted as a result of the MEWS triggering response at a level earlier than in the previous system. We can categorically state that five incidents on the pilot wards, that under the previous system would have led to MET calls, were safely dealt with on the ward (mostly cases of severe post-operative hypotension or cardiac dysrythmias). This would appear to explain the fall in MET calls on our pilot wards while overall hospital MET calls remained static. The MEWS cannot prevent critical illness per se but can, in our opinion, lead to earlier intervention and more rapid treatment, and certainly to a reduction in the number of unnecessary or inappropriate emergency calls.

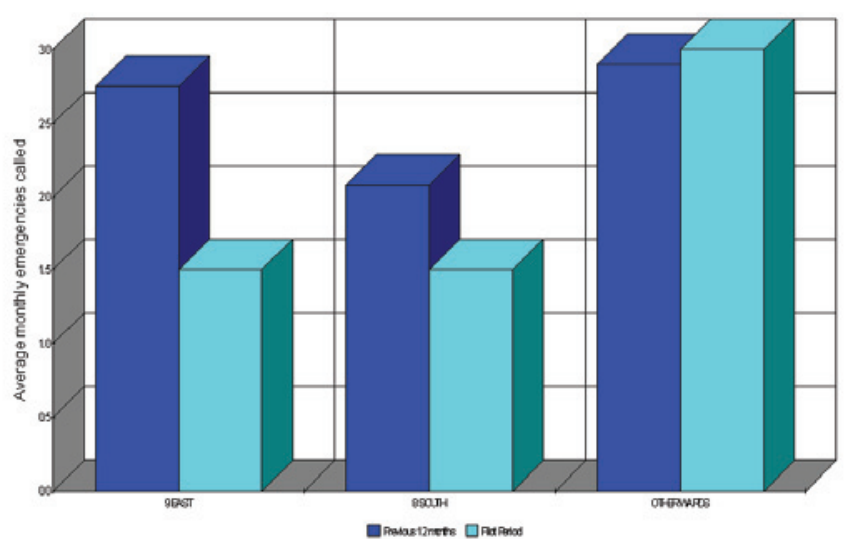

Figure 6. Average monthly MET calls before and during the pilot period

The nurse satisfaction survey results also strongly suggest improved clinical outcomes for patients. We therefore, confidently anticipate that overall hospital MET calls will fall by a (highly) statistically significant number once the MEWS is adopted by all wards. However, this must be tested over a suitably longer period of analysis.

Our averting of (at least) five MET calls on the MEWS wards appears modest (that is, 1.25 per ward per month). Yet, if this average were repeated once the MEWS is rolled out to all seven hospital wards, it could avert 105 MET calls per year, which equates to a $42.7 \%$ reduction. This would represent an enormous reduction in stressful emergencies, hospital costs associated with critical care, and of course significantly improved patient outcomes.

\section{SUMMARY AND CONCLUSIONS}

This paper has described the background to a problem that has been identified in hospitals worldwide. It has outlined the rationale for choosing a MEWS, how we adapted it for use at the Mater Private Hospital, and steps taken before it was successfully rolled out hospital-wide.

Key elements in change management were considered to be the consultation process with stakeholders, piloting the MEWS and testing its effectiveness, training and education opportunities and further research to establish how it might be improved in the future.

The MEWS chart that replaced the previous method of recording observations is clear, concise and simple to use. Our experience suggests a simple change to the way nurses record routine observations can lead to a significant improvement in clinical outcomes for patients and increased satisfaction of both nurses and doctors. The introduction of a MEWS at the Mater Private Hospital has shown similar benefits to those found in many hospitals in the UK and USA who have used MEWS.

Use of MEWS has helped to reduce medical emergencies and ICU admissions on the pilot wards by pre-empting the need for MET calls. It has also been seen to improve multi-disciplinary communication and empower nurses to make better clinical decisions. These factors reduce the nurse's overall workload and stimulate education, training and support initiatives, which enhance the nurse's role as patient advocate. They may aid recruitment and retention.

The role of the CNS was pivotal to this project. The CNS supported ward nurses after hours, but the MEWS provided a theoretical framework underpinning the role of CNS and an optimal referral mechanism for it.

In summary, the pilot demonstrated an edifying trilogy of reduced emergencies, popularity with nurses and doctors, and increased confidence in nurses' self-rated ability to identify and respond to a 
deteriorating patient on the pilot wards. While our pilot was brief in order to avoid seasonal factors, this was sufficient to make a decision to roll out the MEWS system to all wards from February 2008, and to expand the PART to daytime as well as after hours support at some stage in the future. Following the success of the pilot, we are now extending the system to all wards to test its effectiveness across a range of specialties and to more reliably determine its effect on MET calls over an extended time period.

\section{RECOMMENDATIONS}

Further research is needed to evaluate the success of the MEWS in order to modify it further to suit the Mater Private Hospital's unique needs. Research questions, which will help us improve the system include:

- Which are the most reliable indicators of a deteriorating patient (for example, respiratory rate, blood pressure, urine output)?

What are the optimal levels to set the trigger parameters?

- How does the MEWS help nurses make informed choices about sick patients?

We recommend all acute Australian hospitals, especially those without after hours resident medical officer cover, consider adapting a MEWS to their own needs to provide optimal care for critically ill ward patients.

\section{Acknowledgement}

The authors would like to express their sincere thanks to all staff on the pilot wards, 8 South (orthopaedics) and 9 East (neurovascular), for their enthusiastic pioneering of the new system, and to the doctors on those wards and in ICU for their patience and support.

\section{REFERENCES}

Buist MD, Moore GE, Bernard SA, Waxman BP, Anderson JN, Nguyen TV (2002). Effects of a medical emergency team on reduction of incidence of and mortality from unexpected cardiac arrests in hospital: preliminary study. BMJ 324 (7334), 387-390.

Campling EA, Devlin HB, Hoile RW, Lunn JN (1995). The report of the national confidential enquiry into perioperative deaths 1992/93. London, NCEPOD.

Department of Health. (2000). Comprehensive Critical Care Review: A Review of Adult Critical Care services. London, HMSO.

Featherstone P, Smith G, Linnell M, Easton S, Osgood V (2002). Impact of a one-day inter-professional course (ALERT ${ }^{\mathrm{TM}}$ ) on attitudes and confidence in managing critically ill adult patients. Resuscitation 65 (3), 329-336.

Franklin C, Mathew J (1994). Developing strategies to prevent in-hospital cardiac arrest: analysing responses of physicians and nurses in the hours before the event. Critical Care Medicine 22 (2), 244-247.

Goldhill DR (2001). The critically ill: following your MEWS. QJM: monthly journal of the Association of Physicians 94 (10), 507-510.

Goldhill DR, Sumner A (1998). Outcome of intensive care patients in a group of British intensive care units. Critical Care Medicine 26 (8), 1337-1345.

Hadfield J, Bishop G, Flabouris A, Hillman K, Parr M (2000). The medical emergency team: effect on hospital cardiac arrests. Resuscitation 45, S37.

Institute for Healthcare Improvement (2007). Early warning systems: scorecards that save lives. [Online]. Available at: www. ihi.org/IHI/Topics/Critical Care/Improvements/Stories. Accessed 05 May 2007.
Lee A, Bishop G, Hillman KM, Daffurn K (1995). The medical emergency team. Anaesthesia and Intensive Care 23 (2), 183186.

Lunn JN, Devlin HB, Hoile RW (1996). The report of the national confidential enquiry into perioperative deaths 1993/94. London, NCEPOD.

Metcalfe MA, Sloggett A, McPherson K (1997). Mortality among appropriately referred patients refused admission to intensive care units. Lancet 350 (9070), 7-12.

McGloin H, Adam SK, Singer M (1999). Unexpected deaths and referrals to intensive care of patients on general wards. Journal of the Royal College of Physicians 33 (3), 255-259.

McQuillan P, Pilkington S, Allan A, Taylor B, Short A, Morgan G, Nielsen M, Barrett D, Smith G, Collins CH (1998). Confidential inquiry into quality of care before admission to intensive care. BMJ 316 (7148), 1853-1858.

Morgan RJM, Williams F, Wright MM (1997). An Early Warning Scoring System for detecting developing critical illness. Clinical Intensive Care 8 (2), 100.

Odell M, Forster A, Rudman K, Bass F (2002). The critical care outreach service and the early warning system on surgical wards. Nursing in Critical Care 7 (3), 132-135.

Palmer R (2004). Using an early warning system in a medical assessment unit. Nursing Times100 (48), 34-35.

Parissopoulos S, Kotzabassaki S (2005). Critical care outreach and the use of early warning scoring systems: A literature review. ICU and Nursing Web Journal 21, 1-13.

Sax FL, Charlson ME (1987). Medical patients at high risk for catastrophic deterioration. Critical Care Medicine 15 (5), 510-515.

Schein RMH, Hazday N, Pena M, Ruben BH, Sprung CL (1990). Clinical antecedents to in-hospital cardiopulmonary arrest. Chest 98 (6), 1388-1392.

Smith AF, Wood J (1998). Can some in-hospital cardio-pulmonary arrests be prevented? A prospective survey. Resuscitation 37 (3), 133-137.

Sprung CL, Geber D, Eidelman LA, Leonid A, Baras M, Pizov R, Nimrod A, Oppenheim A, Epstein L, Cotev S (1999). Evaluation of triage decisions for intensive care admission. Critical Care Medicine 27 (6),1073-1079.

Stenhouse C, Coates S, Tivey M, Allsop P, Parker T (2000). Prospective evaluation of a modified early warning score to aid earlier detection of patients developing critical illness on a general surgical ward. British Journal of Anaesthesia 84 (5), $663 P$.

Subbe CP, Kruger M, Rutherford P, Gemmel L (2001). Validation of a modified Early Warning Score in medical admissions. QJM: monthly journal of the Association of Physicians 94 (10), 521-526.

Trevett $L$ (2007). Introduction of an Early Warning System. Private Hospitals Association of Queensland, Innovative Practice in the Private Sector Conference, Wesley Hospital, 22 June 2007.

Wallis CB, Davies HTO, Shearer AJ (1997). Why do patients die on general wards after discharge from intensive care units? British Journal of Anaesthesia 52 (1), 9-14.

Walsh M, Ford P (1989). We always do it this way: Rituals in nursing, part 1. Nursing Times 85 (41), 26-28.

Wood J, Smith A (1999). Suboptimal ward care of critically ill patients. Active management should prevent cardiopulmonary arrest. BMJ 318 (7175), 51-52. 